\title{
Concentraciones Plasmáticas de Magnesio en Lactantes con Diarrea Aguda, Deshidratación y Acidosis
}

\author{
Dra. Andrea Gleisner E. ${ }^{1} ;$ B.Q. Gonzalo Soto $\mathrm{T}^{2}{ }^{2}$ : Dr. Guillermo Venegas $\mathrm{V}_{.}{ }^{3}$; \\ Dr. Oscar Solar G. ${ }^{4}$;Q.F. Aldo Rodríguez $\mathrm{E}^{5}$
Plasmatic Magnesium Concentrations in Infants With and Without Acute Diarrhoea Dehydration and Acidosis

Plasma levels of $\mathrm{Mg}$ were determined in a group of 22 healthy infants, resulting in an average of $23.9 \pm 1.63 \mathrm{mg} / \mathrm{l}$. These results are in - keeping with those obtained by foreign authors. A sccond group of infants was made up of 20 patients having acute diarrohea associated with dehydration, acidosis or both. Plasma levels of $\mathrm{Mg}$ in this group were significantly elevated as compared to the control group, with an average of $26,75 \pm 3,49 \mathrm{mg} / \mathrm{l}(\mathrm{p}<0.01)$. These results suggest that early treatment including polyionic solutions with $\mathrm{Mg}$ are not needed in this condition.

(Key words: Serum magnesium concentration. Acute diarrhoea. Healthy infants).

E] magnesio (Mg) ocupa el segundo lugar entre los cationes intracelulares. Es un elemento csencial para la producción y transferencia de energía, para la contractilidad muscular y la excitabilidad neuromuscular $y$ es un cofactor esencial de numerosos sistemas enzimáticos involucrados con otras funciones vitales. Además se relaciona con los otros cationes, por ejemplo Ca, de un modo semejante a la relación que existe entre $\mathrm{Na}$ y $K$. Más aún, hay una relación interdependiente entre la razón intracelular $\log (\mathrm{K}+) /(\mathrm{Na}+)$ con el $\log (\mathrm{Mg}++) /(\mathrm{Ca}++)^{1-6}$.

Las concentraciones normales de $\mathrm{Mg}$ en el plasma oscilan entre 17,01 y $30,37 \mathrm{mg} / \mathrm{l}$ (Tabla 1). En el hombre los requerimientos basales del Mg varían durante el transcurso de la vida, estimándose en el lactante alrededor de 40 a 70 Ing $\mathrm{x}$ día (Tabla 2). Los requerimientos de $\mathrm{Mg}$ durante la alimentación parenteral en nifios oscilan entre 3 y $6 \mathrm{mg} \times \mathrm{kg} \times$ día 10 .

El $\mathrm{Mg}$ es absorbido en el intestino delgado, dependiendo de varios factores, como lá velocidad del tránsito intestinal y algunos elementos

1. Instructor JC Depto. Pediatria, Facultad de Medicina, Universidad de Concepción.

2. Instructor JC Depto. Análisis Instrumental Facultad de Farmacia, Universidad de Concepción

3. Profesor Asociado de Pediatría, Facultad de Medicina, Univetsidad de Concepción.

4. Pediatra, Hospital Guillemo Grant Benavente, Con. cepción.

5. Profesor Asociado. Depto. Análisis Instrumental, Facultad de Fannacia, Universidad de Concepción.
Tabla 1

Niveles Normales de Magnesio Plasmático

\begin{tabular}{|c|c|c|}
\hline Autos & $\begin{array}{l}\text { Mg. PLASM. } \\
\text { (mg./I.) }\end{array}$ & $\begin{array}{l}\text { Catidad de } \\
\text { la muestra }\end{array}$ \\
\hline \multicolumn{3}{|l|}{ Maxwell y } \\
\hline Kleemann (8) & $19,44-25,52$ & Adultos \\
\hline Nelson, Vaugham $y$ & & \\
\hline McKay(12) & $20,05-30,3 ?$ & Niños \\
\hline \multicolumn{3}{|l|}{ Caddell y } \\
\hline Colabs (9) & $18,72-19,44$ & Lactantes 1-6 mese \\
\hline Caddetl J.2.(1) & $17,01-29,16$ & Nin̄os \\
\hline
\end{tabular}

Tabla 2

Requerimientos Diarios de Magnesio (1)

\begin{tabular}{lll}
\hline Recién nacidos & \multicolumn{1}{c}{$10,2 \mathrm{mgr}$} \\
Lactantes & $1-12$ meses & $40.70 \mathrm{mgr}$ \\
Niños & $1-3$ años & $100-150 \mathrm{mgr}$. \\
Niños & 3.12 años & $200-300 \mathrm{mgr}$. \\
& $12-18$ años & $250-400 \mathrm{mgr}$. \\
\hline
\end{tabular}

presentes en la dieta (calcio, fósfóro, grasas, fitatos, proteínas, $V$ it $D)^{1}$. Su excreción se realiza principalmente por via renal, siendo mínima la excreción intestinal (menos de $1,4 \%$ en condjciones normales). El riñón, es capaz de reducir las pérdidas al mínimo (menos de $12 \mathrm{mg} /$ dia) ien casos de escasez provocada por aportes inadecuados o pérdidas exageradas ${ }^{1-3-4}$.

Las manifestaciones clínicas de hipomagnesemia son varias, inespecíficas y de diferente magnitud ${ }^{1 \rightarrow-7-11}$. La escasez aguda de $\mathrm{Mg}$ es más 
severa cuanto menor sea el niño. Sus manifestaciones incluyen hiperexcitabilidad neuromuscular (hasta convulsiones) y arritinias cardiacas, entre otras.

El aporte indiscriminado de soluciones parenterales con $\mathrm{Mg}$ o aún el suministro habitual en presencia dc insuficiencia renal, pueden inducir efectos tóxicos derivados de hipermagnesemia $^{1-1} 1$. Los signos de toxicidad, descritos, van desde depresión de la excitabilidad neuromuscular hasta alteraciones de las funciones cardio. vasculares $y$ respiratorias en las intoxicaciones severas con paro cardíaco e insuficiencia renal respiratoria.

Este estudio se realizó con el doble propósito de contribuir a definir las concentraciones plasmáticas normales de Magnesio en nuestro medio en lactantes $y$ de determinar si en sujetos de tal edad con diarrea aguda y deshidratación existe evidencia de escasez del ion que justifique el emplco de soluciones que lo contengan, en el tratamiento.

\section{METODOS y PACIENTES}

Se midieron las concentraciones plasmáticas de $\mathrm{Mg}$ en 22 lactantes eutróficos sanos (11 de sexo femenino): entre l y 12 meses de edad. seleccionados al azar, entre quienes asistieron al consultorio externo para control habitual de salud $y$ en 20 lactantes ( 9 de sexo femenino). elegidos al azar, entre los que ingresaron al Servicio de Pediatria por diarrea aguda con deshidratación, asociada o no a acidosis. Es. tos lactantes cumplian los mismos requisitos de eutrofia, edad entre 1 y 12 meses y ausencia de otra enfermedad en el momento del ingreso.

En los enfermos las muestras se tomaron al ingresar en el hospital $y$ en los sanos en el momento de consultar, centrifugándolas antes de 30 minutos de haber sido tomadas, usando como anticoagulante citrato de sodio. Posteriormente fueron analizadas en duplicado usando espectrofotómetro Perkin Elmer 308. Para el análisis estadistico se empleó la prueba Dócima " $t$ " de Student.

\section{RESULTADOS}

Las concentraciones de $\mathrm{Mg}$ plasmático obtenidas en el grupo control fueron en pro. medio $23,90 \pm 1,63 \mathrm{mg} \times 1$, rango 21,32 a $27,18 \mathrm{mg} / \mathrm{l}$ (Tabla 1). La distribución de los valores según la edad en este mismo grupo está descrita en la Figura 1.

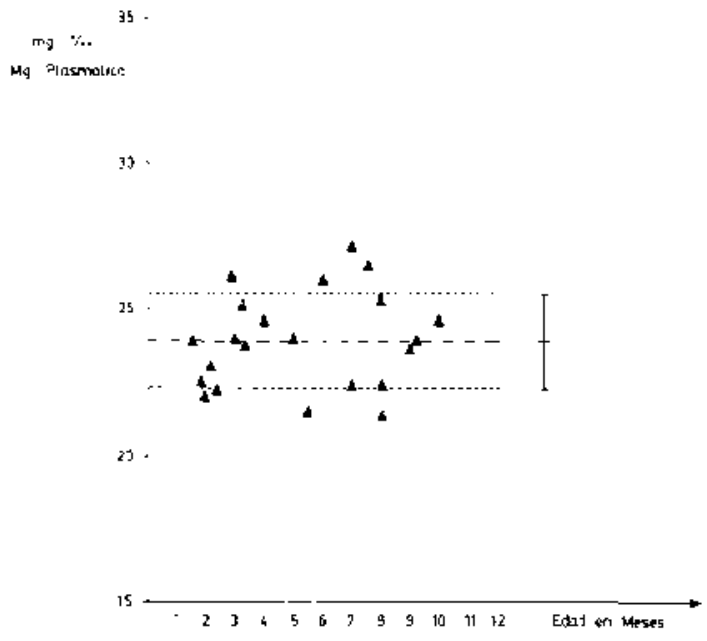

Figura 1. Niveles de Me. plasmático en lactantes sanos.

En la Figura 2 se compara el grupo control con los lactantes con diarrea aguda. de los que 5 tenian además acidosis. La con. centración promedio de $\mathrm{Mg}$ en el plasma de este grupo fue $26,75 \pm 3,49 \mathrm{mg} \times 1$, con un rango entre 20,08 y $33,40 \mathrm{mg} \times \mathrm{I}$. Cabe hacer notar que había mayor dispersión de los valores en los niños con diarrea que en aquellos del grupo control. El promedio exhibido por los enfermos fuc significativamente más alto $(\mathrm{p}<0,01)$ que en los controles.

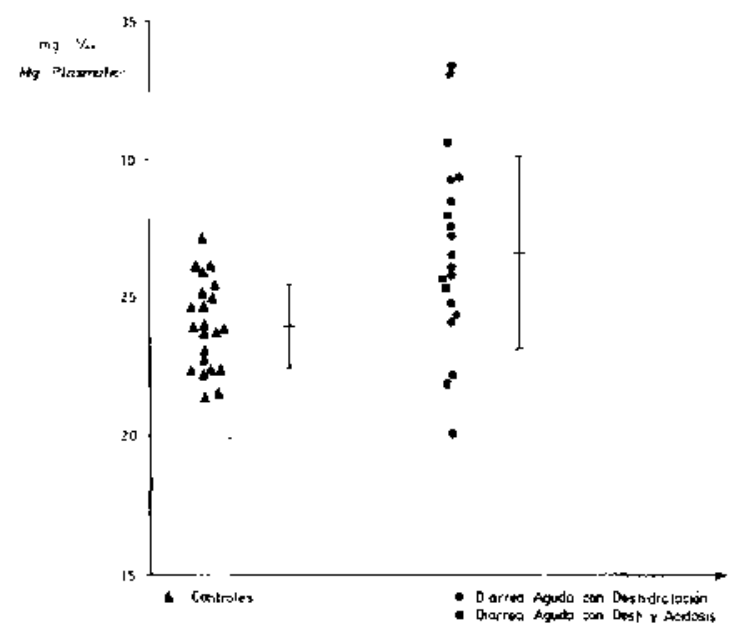

Figura 2. Niveles de Mg. plasmático en lactantes sanos y enlaclantes portadores de un sindrome ayudo con deshidratación y/o acidosis.

\section{DISCUSION}

Las concentraciones plasmáticas de $\mathrm{Mg}$ en nuestros lactantes sanos fueron semejantes a 
las descritas por autores extranjeros ${ }^{1-4-12}$. En los lactantes con diarrea los valores obtenidos fueron significativamente más altos que en el grupo control $(p<0,01)$.

De acuerdo con estos resultados no sería indispensable emplear soluciones parenterales poli-iónicas conteniendo $\mathrm{Mg}$ en el tratamiento inicial de la diarrea aguda con deshidratación en lactantes eutróficos, mientras no se demuestre un déficit de este jón: La administración de $\mathrm{Mg}$ deberia estar así respaldada por la medición de concentraciones plasmáticas una vez reestablecido el balance hidrico.

\section{RESUMEN}

La concentración de $\mathrm{Mg}$ en el plasma de 22 lactantes sanos fue en promedio de 23,90 $\pm 1,63 \mathrm{mg} \times 1$, similar a la de otras publicaciones. En 20 lactantes con diarrea aguda y deshidratación con $o$ sin acidosis, las concentraciones plasmáticas fueron leve pero significativamente elevadas con respecto a los controles sanos, promedio $26,75 \pm 3,49 \mathrm{mg} \times 1$. $(\mathrm{p}<0,01)$.

\section{REFERENCIAS}

1. Caddell, J.L.: Magnesium in the Nutrition of the Child. Clin. Pediatr. 13: 263, 1974.

2. Watson, W.S., Hiditch, T.E., Horton, P.W., Do vies, D.L., and Lindsay R.: Magnesium Me- tabolism in Blood and the Whole Body in Man using $\mathrm{Mg}$ 28. Metabolism, 28: 90, 1979.

3. Burch, G.E., Giles, T.D.: The importance of Magnesium Deficiency in Cardiovascular Disease. Am. Heart. J. 94: 649, 1977.

4. Wacker, $w$. and Parisi, A.: Magnesium Metabolism. New Engl. J. Med. 278: 658, 1968.

5. Whang $R$, Oei T., Aikawa J., Ryan M., Watanabe A., Chrysant $S$. Fryer $A .:$ Magnesium and Potassium Interrelationships Experimental and Clinical. Acta. Med. Scand. Suppl. 647: $139,1980$.

6. Whang, $R$., Oei $T$, Aikewe, J, Watanabe, A., Vannatta, J., Fryer. A., Markanich, $M_{\text {.: Pre- }}$ dictors of Clinical Hypomagnesemia. Arch. Intern. Med. 144: 1794, 1984.

7. Caddell, J.L.: Exploring the Magnesium-deficient Weanling Rat as an Animal Model for the Sudden Infant Death Syndrome: Physical, Biochemical. Electrocardiographic, and Gross Pathologic Changes. Pediat. Res. 12: 1157, 1978.

8. Maxwell, M.H., Kleemann, C.R.: Clínica de Trastornos Hidroelectrolíticos. Ediciones Toray S.A. Barcelona, España. Segunda Edición 1976, Capítulo $9 \mathrm{Pag}$. 33?.

9. Caddell, I.L., Byrne, P.A., Triska, R.A. and Mc Elfresh, A.E.: Cortelation of Clinical and Laboralory Data in Infants from one to six Months of Age. Clin. Pediatr. 14: 478, 1975.

10. Kanarek, K.S., Willioms, P.R. and Curran J.S.: Total Parenteral nutrition in lnfants and Children. Adv. Pediatr. 29: 151, 1982.

11. Wong $E$., Rude $R$., Singer $F$, Shaw S.T.: A high prevalence of hyponagnesemia and hypermagnesemia in hospitalized patiens. Am. J. Clin. Pathol. 79: 348, 1983.

12. Yelson, Vaughon, Mc Kay.: Eds. Tratado de Pediatria. Traducción de la 9a. Edición Norteamericand. Barcelona, 1971, Salvat. 